\title{
Review
}

\section{Viral capsids: Mechanical characteristics, genome packaging and delivery mechanisms}

\author{
W. H. Roos ${ }^{\text {a }}$ I. L. Ivanovska ${ }^{\text {a }, ~ A . ~ E v i l e v i t c h ~}{ }^{\text {b }}$ and G. J. L. Wuite ${ }^{\text {a * }}$ \\ ${ }^{a}$ Fysica van complexe systemen, Vrije Universiteit, 1081 HV Amsterdam (The Netherlands), \\ Fax:+31205987991, e-mail: gwuite@nat.vu.nl \\ ${ }^{\mathrm{b}}$ Department of Biochemistry, Centre for Chemistry and Chemical Engineering, Lund University, P.O. Box 124, \\ 22100 Lund (Sweden)
}

Received 14 October 2006; received after revision 18 December 2006; accepted 27 February 2007

Online First 19 March 2007

\begin{abstract}
The main functions of viral capsids are to protect, transport and deliver their genome. The mechanical properties of capsids are supposed to be adapted to these tasks. Bacteriophage capsids also need to withstand the high pressures the DNA is exerting onto it as a result of the DNA packaging and its consequent confinement within the capsid. It is proposed that this pressure helps driving the genome into the host, but other mechanisms also seem to play
\end{abstract}

an important role in ejection. DNA packaging and ejection strategies are obviously dependent on the mechanical properties of the capsid. This review focuses on the mechanical properties of viral capsids in general and the elucidation of the biophysical aspects of genome packaging mechanisms and genome delivery processes of double-stranded DNA bacteriophages in particular.

Keywords. Capsid mechanics, genome packaging, genome delivery, osmotic pressure, atomic force microscopy, optical tweezers, biophysics.

\section{Introduction}

Viral capsids are nanometre-sized containers that possess complex mechanical properties and whose main function is to encapsidate the viral genome in one host, to transport it and to subsequently release it inside another host cell. One of the remarkable properties is the self-assembly of capsids in a regular and well-defined structure. These self-assembled capsids need to protect effectively the packaged genome, whereas it also needs to be opened at the right place and time to release the genome. Therefore,

* Corresponding author. the demands on the capsids mechanics and function are highly specific.

Bulk chemical assays as well as electron microscopy and crystallography experiments have been used to study viral systems over many years and a wealth of information is extruded from such experiments. In the last few years single-particle/molecule techniques [1] have also become available to study (i) mechanical properties of viral capsids [2], (ii) phage genome packaging [3], and (iii) phage genome ejection [4]. This review gives an overview of research done on these three topics and the relation between these topics is highlighted. Experiments using these techniques are discussed with an emphasis on biophysical methods. 


\section{Capsid mechanics}

The mechanical properties of viral capsids are crucial for its proper functioning, because rupture of the capsid due to defects occurring during maturation or as a result of external influences outside the host will in general result in a loss of infectivity. Questions that rise when considering mechanics of capsids are, among others, how do the mechanical properties change during maturation, what is the difference in properties of empty vs. full capsids, what is the difference between spherical and cylindrical capsids and, in view of their different infection strategies, how do the mechanical properties of animal, plant and bacterial viruses relate to each other?

Bulk measurements have yielded insight into the 'averaged' properties of viruses; however, by introducing single-particle techniques such as atomic force microscopy (AFM) [5], it became possible to measure and manipulate individual viruses. AFM has been used to apply point forces on single particles [2, 6-8], whereby ultimately the strength of the protein-protein interactions in the capsid is measured. Viruses can be imaged repeatedly in a non-destructive way using this technique. Furthermore, AFM is uniquely suited to study mechanical properties of small biological entities such as viruses due to its high sensitivity in applying and measuring forces combined with the possibility to work under physiological conditions. AFM has been used to image a range of viruses [9-13], but here the focus is on those experiments measuring the mechanical properties of viral capsids.

A linear elastic response is expected for indentations of thin homogeneous shells when the indentation is in the order of the shell thickness [14]. The shell spring constant $k_{\text {shell }}$ is given by

$k_{\text {shell }}=\alpha E h^{2} / R$

with a geometry-dependent proportionality factor $\alpha$, Young's modulus $E$, the shell thickness $h$ and the radius $R$ [14]. The value of $E$ can be calculated when $k_{\text {shell }}$ is measured and when values for $h, R$ and $\alpha$ are available, for instance from cryo-electron microscopy studies, X-ray crystallography and theory. For a small deformation of a thin-shelled capsid it is possible to calculate the proportionality factor $\alpha$ analytically by modelling the capsid as a homogeneous, spherical and elastic shell. In the case of large deformations or for viruses with thick shells, a finite-element analysis can be performed to model the elastic behaviour of these viruses. Such analyses have, for instance, shown that for large indentations of the (thick) shell of cowpea chlorotic mottle virus (CCMV), the deformation remains linear with the force and that Eq. (1) still holds $[6,15]$. The proportionality factor $\alpha$ can then be extracted from the finite-element analysis [6]. Typical values for $\alpha$ are around unity $[2,6]$.

Young's modulus can differ significantly between different viruses. AFM experiments on the Bacillus subtilis bacteriophage $\Phi 29$ [2] have shown that Young's modulus of the immature, empty $\Phi 29$ prohead (procapsid) is $\sim 1.8 \mathrm{GPa}$, which is comparable to other structural proteins like actin, tubulin and collagen [16] and to that of hard plastics. Comparing Young's modulus of the $\Phi 29$ prohead with the empty capsid of the plant virus CCMV, which is $0.14 \mathrm{GPa}$ [6], and with the immature capsid of the animal virus murine leukemia virus (MLV), which is $0.23 \mathrm{GPa}$ [7], it was found that the latter two are one order of magnitude smaller than the former. The differences in the mechanical characteristics of $\Phi 29$ and those of CCMV and MLV seem to be reflected in their assembly pathways. The capsid of phage $\$ 29$ selfassembles and subsequently a packaging motor is required for DNA encapsidation. The internal pressure that the packaged DNA exerts onto the $\Phi 29$ capsid is approximately $6 \mathrm{MPa}(60 \mathrm{~atm})$ [3]. CCMV and MLV also self-assemble, but during this process its RNA is integrated into the capsid. Therefore, it is unlikely that the RNA would exert high pressures onto the viral shell and consequently the mechanical requirements on their capsids should be lower than for $\Phi 29$.

The indentation experiments on $\Phi 29$ proheads and CCMV capsids show that for both types of particles a reversible linear regime was observed when the indentation did not exceed $20-30 \%$ of capsid height. For higher indentations a catastrophic drop in the force was observed, which is attributed to irreversible deformation of the capsid. Comparing the threshold value for nonlinear deformation of empty $\$ 29$ proheads with that of empty CCMV capsids shows that the latter has a threshold force of $0.60 \pm 0.04 \mathrm{nN}( \pm$ standard error of mean, SEM) [6], whereas the former can withstand several nanonewtons. Only for forces above $2.8 \pm 0.3 \mathrm{nN}$ ( \pm standard deviation, $\mathrm{SD}$; $\mathrm{n}=11$ ) [2] does the response of the $\Phi 29$ capsid starts to deviate from linearity. Note that the experiments on $\Phi 29$ have been performed with higher loading rates than those on CCMV. Repeating the experiments with identical loading rates might result in a smaller difference between these two viruses. Even though the $\$ 29$ experiments were not performed on mature viruses, but on empty proheads, these results give an indication that bacteriophage capsids will be able to withstand higher pressures than plant viral capsids. This is also what one expects in view of their different genome encapsidation mechanisms as discussed before. Figure 1 shows an AFM image of an indented $\Phi 29$ prohead. 
To test whether there is a difference in mechanical properties of empty and full capsids, experiments on the plant virus CCMV [6] and on the animal virus minute virus of mice (MVM) [8] have been performed. The experiments on CCMV show that empty capsids as well as those containing the RNA genome are highly elastic. As for empty capsids, also the full capsids behave linearly for indentations of up to $20-30 \%$ in capsid height. The threshold force for irreversible deformation was observed at essentially the same deformation for all of the CCMV capsids, but the full capsid is less elastic than empty ones. This means that a full capsid requires a higher threshold force for irreversible deformation and this force turns out to be proportional to the spring constant. The measured values for the spring constant and the threshold force for empty capsids are $0.15 \pm 0.01 \mathrm{~N} / \mathrm{m}( \pm \mathrm{SEM})$ and $0.60 \pm 0.04 \mathrm{nN}$, respectively, and for full capsids $0.20 \pm 0.02 \mathrm{~N} / \mathrm{m}$ and $0.81 \pm 0.04 \mathrm{nN}$, respectively. The effect of a single point mutation of the CCMV capsid was tested using a salt-stable mutant. Compared to the full, wild-type (wt) capsid, the full mutant capsid had a $50 \%$ higher spring constant and a $30 \%$ higher threshold force for irreversible deformation. This impressive effect of a single point mutation on the capsid strength is valuable information when one wants to make strong nanocontainers, and these results could be used for CCMV as these capsids are already used as a container for nongenomic material [17].

The experiments on the parvovirus MVM capsids reveal that the packaged genome can induce an anisotropic stiffness of the viral capsid [8]. Whereas the empty capsid has an isotropic spring constant of $0.6 \pm 0.1 \mathrm{~N} / \mathrm{m}$, the DNA containing virion has an anisotropic spring constant. This depends on the symmetry axis along which is measured. The spring constant along the five-, three-, and twofold symmetry axes is $0.6 \pm 0.2,0.8 \pm 0.4$ and $1.4 \pm 0.5 \mathrm{~N} / \mathrm{m}$, respectively. Finite-element modelling indicates that this anisotropic reinforcement of the capsid could be mediated by the attachment of the genome at 60 concavities inside the capsid. In general, it is expected that the spring constant of an icosahedron depends on the symmetry axis along which is measured. However, finite element modelling shows that for a certain ratio of the shell thickness over the radius the spring constant along the two-, three- and fivefold axes coincides [8]. This is the case for the empty MVM capsid and therefore it shows an isotropic spring constant. The experiments on CCMV did not show a spring constant dependency on its symmetry axes either [6]. A possible explanation is that the varying shell thickness of the CCMV capsid results in a

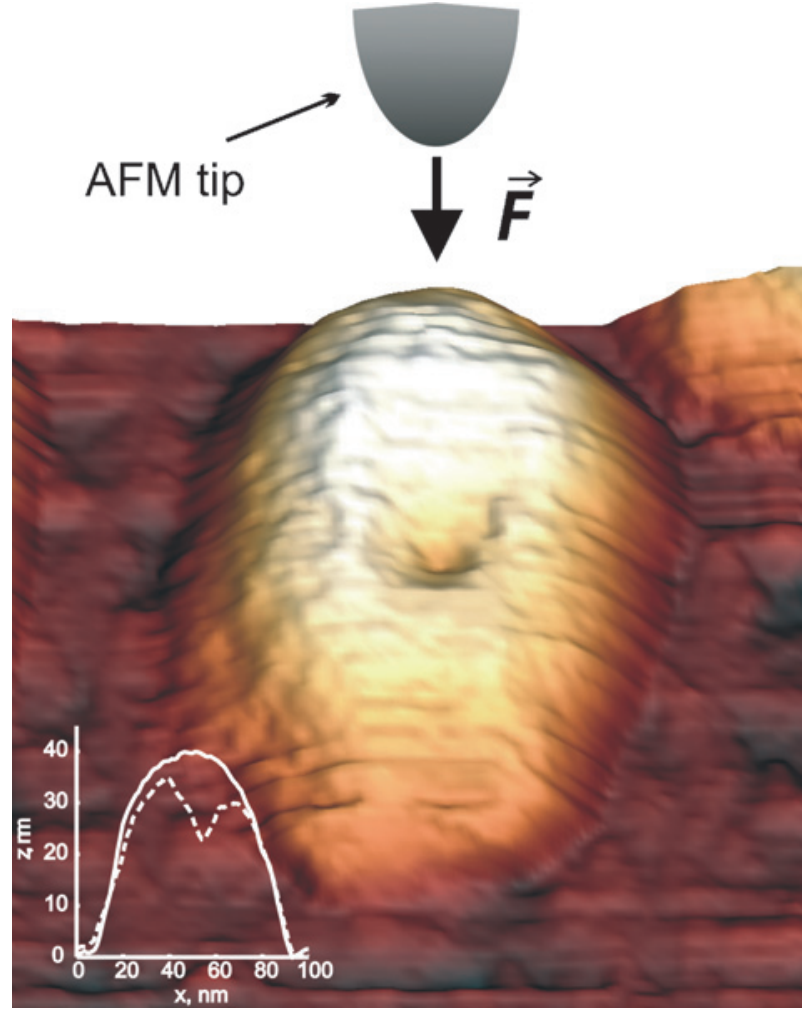

Figure 1. Indentation experiments on phage $\Phi 29$ [2]. Atomic force microscopy (AFM) image of an empty capsid with an AFM tip drawn to scale. In the middle of the virus the indentation left by the tip can be observed. Inset: Profile of the prohead before (solid line) and after (dotted line) the indentation.

uniform spring constant along the different symmetry axes. Furthermore, it is also possible that the differences in spring constant were just not resolved; more measurements could clarify this question.

The effects of maturation on the mechanical properties of MLV, a retrovirus that matures after budding, have also been tested by AFM measurements [7]. During maturation, which is a prerequisite for infectivity [18], the virus profoundly changes its morphology, but not its diameter, as observed by electron microscopy $[19,20]$. There is also a pronounced difference in the stiffness before and after maturation [7]. The immature shell has a spring constant of $0.68 \mathrm{~N} / \mathrm{m}$, whereas for the mature shell this is more than twofold lower $(0.31 \mathrm{~N} / \mathrm{m})$. Interestingly, finite-element simulations indicate that Young's modulus of mature MLV viruses is $1.0 \mathrm{GPa}$, which is approximately four times higher than that of the immature form $(0.23 \mathrm{GPa})$. Such a difference can be explained by regarding Eq. (1) and considering the fact that the viral shell decreases its thickness from 20 to $4 \mathrm{~nm}$ during maturation. This makes it plausible that $E$ has to increase during maturation, otherwise the overall mechanical struc- 
ture of the virus would be weakened dramatically. In view of the difference in Young's modulus of MLV before and after maturation, it will be interesting to repeat the experiments on immature bacteriophages [2] with mature ones to compare it with the results on MLV capsids.

The spring constants of the capsids of $\Phi 29$, MLV, CCMV and MVM lie within one order of magnitude of each other and some interesting features are observed. A bimodal distribution of spring constants was found across the equatorial region of empty $\$ 29$ capsids, with values of $0.16 \pm 0.04 \mathrm{~N} / \mathrm{m}( \pm$ SD) and $0.31 \pm 0.03 \mathrm{~N} / \mathrm{m}$ on average. This indicates the existence of locally soft regions across the surface of the capsid. The origin of this bimodal distribution within the equatorial region remains unknown; a possible candidate for these softer spots is the particular subunit organisation across the equatorial area. The other studied, empty viral particles did not show a multimodal stiffness distribution over their surface. Theoretical studies on pentameric and hexameric substructures in CCMV capsids give contradicting results. On the one hand, normal mode analysis indicates that pentameric substructures are more flexible than hexameric ones [21]; on the other hand, using a percolation rigidity method, it is argued that pentameric substructures are stiffer [22]. Further theoretical work shows that the local pressure and the radial stress in pentamers should be higher than in hexamers [23]. As no multimodal stiffness distribution was found for CCMV during the AFM measurements, no decisive conclusion can be drawn other than that the pentameric, hexameric and possible other substructures posses a similar flexibility. Measurements with sharper tips could reveal whether there is really any difference.

AFM experiments on the rod-shaped plant virus tobacco mosaic virus (TMV) yield a Young's modulus of $1 \mathrm{GPa}$ [24]. However, as the measurements on TMV are performed in air and because Young's modulus is obtained in an indirect way (via surface friction), it is difficult to compare these results to the previously discussed results of virus indentation experiments. Experiments are also conducted to measure the mechanical stability of TMV capsids in air by exerting lateral forces with an AFM tip [25]. A permanent decrease in height occurred for lateral tip forces $>30 \mathrm{nN}$. These values for the lateral threshold force for irreversible deformation of TMV are again difficult to relate to the threshold force for $\Phi 29$ [2] and CCMV [6] as the latter two values are obtained in liquid and by exerting a force perpendicular to the surface. It was also shown that under weak adsorption conditions, i.e. hydrophobic surfaces, TMV retains a cylindrical cross section of $18 \mathrm{~nm}$, whereas under stronger adsorption, i.e. on hydrophilic surfaces, the virus becomes deformed due to a maximisation of the number of interacting groups.

A complementary method to AFM measurements for testing the mechanical properties of viral capsids is performing experiments on capsid rupturing with osmotic shock or by heat treatment. Using the latter method, it was shown that the pentamers of the phage P22 procapsid are released after a 15-min treatment at $70^{\circ} \mathrm{C}$ [26]. The osmotic shock experiments are performed by incubating DNA-filled viruses in high salt solutions followed by rapid dilution. When the viral shell is less permeable to salt ions than to water, the result of dilution will be an uptake of water and consequent bursting. It is shown that T2, T4 and T6 phage are susceptible to rupture, whereas T1, T3, T5 and T7 remain intact when exposed to the same treatment [27]. This is explained by assuming that the odd-numbered T-phages are more permeable to salt ions than the even-numbered T-phages. Theoretical considerations on the salt concentration inside the DNA filled-capsids show that viruses with a low ion permeability were subject to pressure differences of 10.5 $\mathrm{MPa}$, which resulted in rupture [28]. Those capsids that were more permeable to ions and that did not rupture experienced a pressure difference of 7.4 MPa. Apparently, phages can stand a pressure difference of $7 \mathrm{MPa}$. A different analysis, which considers the viral monomeric interactions, leads to a capsid dimension-dependent rupture pressure of minimally $11 \mathrm{MPa}$ [29]. This is in agreement with the values stated above.

The large difference in capsid strength between, on the one hand, bacteriophages and, on the other hand, plant and animal viruses seems to stem from the high pressures that are present in phages. Phages need this pressure for infecting a new host and this pressure is achieved by a powerful packaging motor, which internalises the genome. In the following section this mechanism of phage packaging is discussed.

\section{Genome packaging}

The capsid of double-stranded (ds) DNA phages readily self-assembles, sometimes in the presence of scaffolding proteins, but in the absence of nucleic acids. Subsequently, a packaging motor internalises its genome (reviewed and discussed in [30-34]), as is also the case for dsRNA bacteriophages [35]. The selfassembly and packaging process can be reproduced in vitro using extracts from infected hosts as shown for instance for phage $\lambda[36-38]$ and phage $\Phi 29[39,40]$. Infectious $\Phi 29$ virions can also be constructed from cloned gene products and synthetic nucleic acids [41] 
and infectious phage $\lambda$ virions have been successfully produced from purified components [42]. During the packaging process, the genome, which can be many microns long, needs to be compressed to fit inside a capsid whose diameter is hundreds of times smaller. Electrostatic repulsion between the nucleic acid strands will hinder the compaction of the genome significantly. Moreover, extensive bending energies need to be overcome as the persistence length of dsDNA and dsRNA is comparable to the diameter of the capsid [29, 43, 44]. In general, however, electrostatic repulsion dominates over bending energy and will give the primary contribution to the internal force built-up [45, 46]. A third factor influencing the packaging is the reduction of entropy of the system. As a result of these energetically and entropically unfavourable changes, it is likely that high forces are involved in the packaging of dsDNA or dsRNA inside phage capsids. The energy needed for this force generation comes from ATP consumption and approximately one ATP molecule is hydrolysed for every two base-pairs that are packaged [47].

DNA in wt bacteriophage $\lambda$ is packaged tightly, as shown in small angle X-ray scattering studies [48]. Subsequent experiments on repulsive forces between dsDNA molecules have led to an estimation of the pressure in phages at such packaging densities to be several $\mathrm{MPa}$, depending on DNA density and salt conditions [49]. It was postulated that this pressure built-up is necessary for ejection of the DNA into the host at the beginning of infection [30].

$\mathrm{X}$-ray studies on the cationic-induced condensation of DNA [50] revealed that the packing density of DNA in vitro is comparable to that in phages [51]. The structure of such condensed DNA resembles that of the DNA in viruses. This suggests that the pressure in capsids is not significant when the appropriate counterions are present. However, small differences in packaging density might have a big effect. Cryoelectron microscopy experiments have shown that the T7 genome is packaged in a spool-like configuration inside the capsid with an average interchain separation of $2.5 \mathrm{~nm}$ [52], whereas this separation for phage $\lambda$ and phage P22 is $\sim 2.4 \mathrm{~nm}$, as derived from X-ray diffraction measurements [48]. The interstrand distance of condensed DNA structures in solution is, depending on the cationic compound, $\sim 2.7-3.5 \mathrm{~nm}$ $[44,50,53]$. This small but significant difference with the DNA spacing in phages might result in a significant pressure being present inside packaged phages. It has been shown theoretically that increasing the interstrand distance from 2.4 to $2.8 \mathrm{~nm}$ results in a significant drop in the ejection force, which is supposed to be positively correlated to the pressure inside the capsid [54]. Measurements on the pressure inside wt phages and inside phages with a bigger DNA interchain distance, which can be accomplished by packaging a shorter genome, could resolve the question whether the close packaging of DNA in phages really results in an elevated pressure. These measurements could be done with AFM indentation experiments on phage $\mathrm{T} 7$ or phage $\lambda$, for which structural studies on the packaging of wt genome and genomes with a shorter length are available [48, 52].

Packaging of the phage $\$ 29$ dsDNA (complexed with gp3) into its procapsid requires a powerful molecular motor [3]. Essential components of this motor are the head-tail connector, the prohead (or packaging) RNA (pRNA) and an ATPase (gp16) [41]. The pRNA attaches in a multimeric complex to the connector; however, it is shown that multimerisation is not essential for connector binding [55]. Whereas pRNA is bound to the procapsid with its central domain, it also has binding sites for gp16 [56]. It is suggested that gp16 serves as a linkage between pRNA and the translocated DNA and that it makes essential DNA contacts during this process [56]. Complementing experiments on \$29 DNA packaging show that packaging of DNA with a nick is as efficient as packaging of nick-free DNA [57]. However, DNA possessing single-strand gaps is only packaged until the first gap. These results indicate that the DNA packaging machinery is not hindered by nicks, but that it does require the two strands of the DNA for uninterrupted packaging.

DNA packaging of $\Phi 29$ takes place through a channel inside the connector. X-ray crystallography imaging revealed that this channel has a diameter of $35 \AA$ at it narrowest part $[58,59]$. The average diameter of dsDNA in its B-form is $23 \AA$, thus it is small enough to pass the channel. The channel wall is largely electronegative, but it includes two electropositive lysine rings. This means that during translocation the negatively charged DNA can interact electrostatically at a few points with the inner connector wall. It turns out that this connector wall can undergo structural changes: imaging of the connector in the pre- and post-injection state suggests that premature ejection of the genome is blocked by gp3, which is present inside an expanded connector at the end of packaging [60].

Using laser tweezers [61], the packaging activity of a single complex in real-time has been followed with unprecedented detail [3]. Here the force the $\$ 29$ portal motor generates, while packaging dsDNA into the capsid, is measured. A biotin-labelled $\Phi 29-\lambda$ DNA construct with a length 1.8 times that of the $\Phi 29$ genome was used to study the packaging. The average packaging time of $6.6 \mu \mathrm{m}$ of DNA, equal to the length of the $\Phi 29$ genome, was $5.5 \mathrm{~min}$. Moreover, it is 
possible to package up to $\sim 5 \%$ more DNA into the phage head than the $\Phi 29$ genome length. Slipping does occur occasionally during packaging, but the motor quickly gets hold of the DNA and packaging continues immediately. Furthermore, it was observed that regular pauses of a few seconds occur during packaging, but no slipping is measured at these pauses. Figure 2 shows a schematic image of the experimental set-up of this experiment.
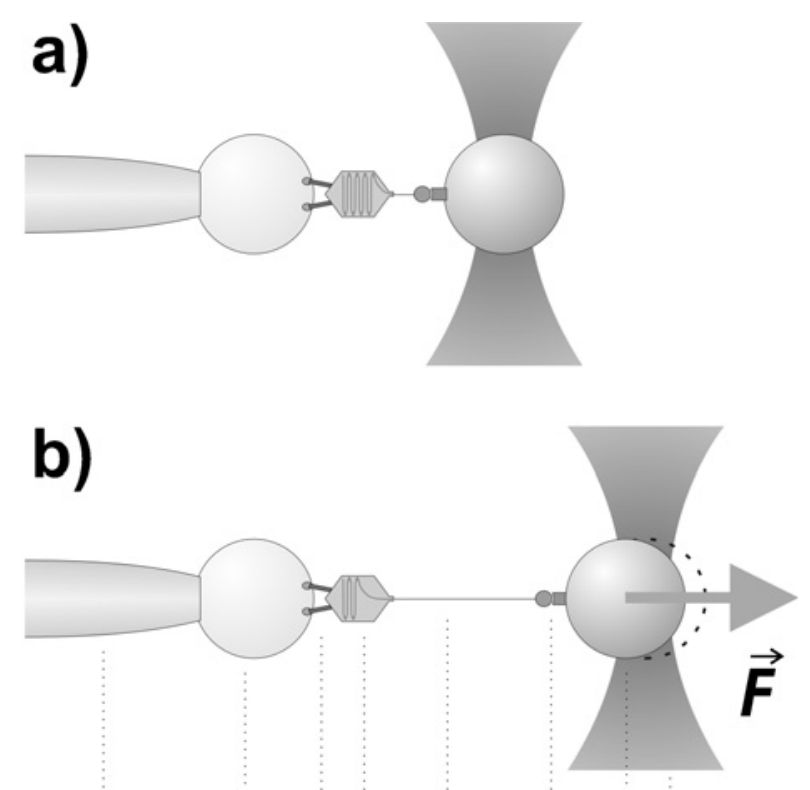

pipette, bead, linker, DNA, linker, bead capsid, optical trap

Figure 2. Schematic of DNA packaging experiment of phage $\$ 29$ [3]. (a) In the "constant force feed-back" mode the bead follows the packaged DNA. This mode allows to measure packaging time and to quantify slipping events. (b) There is no feed-back here and this mode is used to measure the stall force of the packaging motor. Lengths not to scale, images after [3].

It is shown that high forces are needed to package the micrometer-sized DNA into the $\Phi 29$ capsid. The stall force of the packaging motor is $\sim 57 \mathrm{pN}$, which means that it is among the strongest molecular motors known. It can generate forces that are $\sim 2$ times higher than RNA and DNA polymerase [62, 63], 10 times higher than conventional kinesins [64] and 20 times higher than myosin $\mathrm{V}$ [65]. These packaging forces have to overcome extensive electrostatic, elastic and entropic energies to bend the DNA $[44,45]$. The result is that the viral capsids are under a high pressure. Dividing the internal force built-up $(\sim 50 \mathrm{pN})$ by the hexagonal capsid surface area of phage $\Phi 29$, an internal pressure of $\sim 6 \mathrm{MPa}(=60 \mathrm{~atm})$ is estimated. Smith et al. [3] related the work done by the motor during packaging with theoretical calculations of the equilibrium free energy change when DNA is compacted to such liquid crystalline densities as are found inside full capsids. Those predictions are comparable to the estimate of total work done, which indicates that dissipation is not dominant. This means that a significant amount of work done by the motor is reversible. Dissipative effects, however, do play a role during packaging, which is also shown by stochastic rotation dynamics simulations [66].

A detailed mechanochemical cycle of the $\$ 29$ packaging motor was obtained recently [67]. This description of a DNA translocating ATPase follows from the combined results of force-measuring optical tweezers experiments and bulk biochemical assays. It is shown that DNA translocation is not taking place during ATP binding, but probably at the release of phosphate. Furthermore, the motor subunits seem to work in a coordinated fashion with a high processivity.

Phage $\Phi 29$ is an example of a phage that packages non-concatemeric DNA: a linear mature gp3-terminated dsDNA molecule [32]. A different packaging mechanism is followed by phage $\lambda$. This phage packages its concatemeric DNA until a specific sequence (cohesive end sequence, or cos site) is recognised, upon which the DNA is cleaved by the terminase complex, which for phage $\lambda$ is a ring-formed tetramer [68]. Phage T4 (the structure and morphogenesis of which with regard to assembly and DNA packaging and ejection is reviewed in [69]) and phage P22 are examples of viruses that use a headful packaging mechanism. In such a mechanism cleavage of the concatemeric DNA and the termination of packaging takes place after a certain genome density is reached inside the capsid. It was proposed that the P22 portal serves as a sensor to detect that the head is filled up with DNA [70]. Recent work on a high-resolution structure of the P22 capsid, including its portal, has shown that the portal can change its conformation [71]. This seems to happen when a certain pressure inside the capsid is reached due to the packaging of DNA at high densities. Therefore, the portal of phages that use a headful packaging mechanism is likely to act as a pressure-sensitive trigger to initiate the termination of packaging.

The experiments on $\Phi 29, \mathrm{P} 22$ and $\lambda$ have improved our knowledge of packaging mechanisms significantly. However, despite the present information, there are still many open questions regarding the details of how the genome packaging motor in tailed dsDNA phages functions. For instance, it is not clear whether it is a rotary motor or not. DNA translocation takes place at one of the capsid vertices. This portal vertex, which is also the place where procapsid assembly is initiated [31], has a 5-fold rotational symmetry, whereas the attached tail complex has 6-fold (or 12-fold) symme- 
try. Initially, this was regarded as an architectural paradox. Hendrix [72], however, proposed that this symmetry mismatch is essential and reflects the necessity of rotation of a part of the specialised vertex during DNA packaging.

More recently, cryo-electron microscopy experiments on the $\Phi 29$ head-tail connector within the virus [73] and X-ray crystallography experiments on the $\$ 29$ packaging motor [58] confirm the symmetry mismatch and it is again suggested that the packaging motor is a rotary motor. However, fusion of a portal protein with an external shell protein of phage T4, which forms a block to portal rotation during DNA packaging, did not hinder genome packaging [74]. This supports the idea that portal rotation is not necessary for genome packaging [75]. In general, it is shown that a motorratchet mechanism without rotation as well as a rotating motor can explain the data that are available on packaging motors [76]. Thus, it remains an open question what the exact mechanism of DNA packaging is.

Not only bacteriophages package DNA into small capsids, the human infecting herpes simplex virus type 1 (HSV-1) has the same method of genome encapsidation [77]. Whereas the exact molecular mechanism remains elusive, it is at least known which genes are essential for packaging [78, 79]. Furthermore, the packaged HSV-1 DNA possesses a liquid-crystalline density as is also the case for packaged phage genomes [80]. Interestingly, HSV-1 also resembles phages in its capsid assembly [78] and genome delivery system [81, 82]. Analysis of capsid protein structures of different viruses supports the idea of common ancestry of herpesviruses and tailed dsDNA bacteriophages [83, 84], which would explain the similarities.

\section{Genome ejection}

In 1952, Hershey and Chase [85] reported experiments with bacteriophage T2 of which either the DNA component or the protein component was labelled radioactively. With this system it was shown that during T2 infection the capsid stays outside the host cell, whereas the DNA is being injected into the host. It has turned out that other bacteriophages use the same method of delivering their genome into host cells. Furthermore, HSV-1 uses a comparable method in which the capsid remains in the cytoplasm, whereas the genome is transferred into the nucleus [81, 82].

There are large differences in genome length of the different bacteriophages. However, the concentrations at which DNA is packaged is relatively constant, namely $\sim 500 \mathrm{mg} / \mathrm{ml}$ [30]. Clearly, this means that capsid volume is related to genome length. Comparing bacteriophages with viruses that infect eukaryotic cells, it is observed that the genome packaging densities in the former are significantly larger than in the latter [86]. This might also have impact on the pressures inside the capsids and it probably reflects the infection strategies. During eukaryotic viral infection, both the genome and the capsid are transferred together into the host, whereas bacteriophages only inject their genome through the bacterial membrane, leaving the capsid outside [86]. Pressure builtup in the phage capsid could provide the energy for DNA ejection out of it, without ATP hydrolysis or other external energy sources playing a role $[30,54]$. In vitro experiments on DNA ejection out of phage $\lambda$ have shown that ejection can be inhibited by increasing the external osmotic pressure [87]. The direct conclusion from these experiments is that in vitro ejection seems pressure driven.

In vivo phage genome ejection takes place after it is attached to the host cell and a specific receptor protein (present in the bacterial outer membrane) triggers the opening of the capsid. Such capsids can also be opened in vitro by these receptor proteins and opening is followed by ejection of the genome, as shown for instance for phage $\lambda$ [88]. When this is done in aqueous solutions, almost all of its DNA will be expelled. Viral capsids are in general permeable to water and to salt ions [27, 28, 89], which means that in a simple buffer solution, osmotic equilibrium is maintained between the capsid and the buffer solution and that there is no difference in hydrostatic pressure. Therefore, the pressure difference inside and outside of a capsid in a buffer solution containing only small ions is the result of the confinement of the DNA. However, in the in vivo situation, the DNA is not ejected into a simple salt solution, but into the cell cytoplasm. This is a highly concentrated colloidal suspension, characterised by high concentrations of proteins and other macromolecular structures. The ejection of DNA out of the viral capsid will be hindered by such a solution $[87,90]$. When a pressure difference is the sole driving force of the DNA translation, it will drop monotonically during ejection, as a result of a decreased pressure inside the capsid. Therefore there should be a break-even point between the pressure inside and outside. The result is a viral capsid with partially ejected DNA when ejection takes place into a solution with a high osmotic pressure.

Bulk in vitro experiments in polyethylene glycol (PEG) solutions with a phage $\lambda$ mutant having a genome length of $86 \%$ as compared to wt phage $\lambda$ have shown that, for an external osmotic pressure comparable to that of the cytoplasm of host bacteria, DNA ejection is inhibited after half of the genome is 

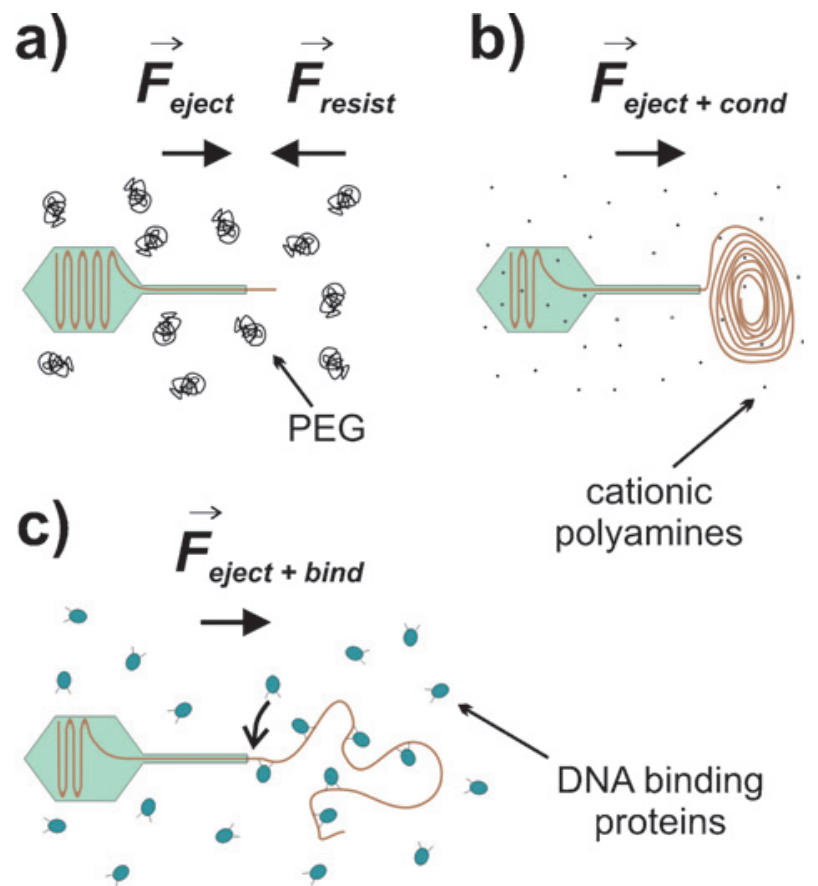

Figure 3. Schematics of a selection of genome delivery processes (a) A high osmotic pressure in the external environment acts as a resisting force to DNA ejection and a part of the DNA will remain inside the capsid $[54,87]$. This is an indication that ejection is (partially) a pressure-driven process. Image after [54]. (b) In the presence of multivalent cationic polyamines the ejected part of the genome condenses. This generates an additional force, pulling the DNA completely out of the capsid [89,97]. (c) Upon attachment of DNA binding proteins to the genome, an additional force is generated that pulls the DNA completely out of the capsid [46, 89]. The shown schematics are intermediate states. The final state in $(a)$ will be partial ejection and the final states of $(b)$ and $(c)$ will be total ejection of the DNA. Images not to scale.

ejected [87,91]. A schematic of this experiment is shown in Figure 3a. A further increase in external osmotic pressure results in a total inhibition of DNA ejection. Additionally, it was revealed that the ejection force is significantly influenced by the genome length inside the capsid. Using a phage $\lambda$ mutant with the same genome length wt phage $\lambda$ and a $78 \%$ genome length mutant, the osmotic pressure that totally inhibits ejection was determined [92]. Together with the results on the $86 \%$ genome length mutant [87], it was shown that inhibition pressures increase with increasing genome length, which is to be expected for a force-driven ejection mechanism dependent on internal capsid pressure. These experiments and the assumption that the osmotic pressure of $E$. coli is at least $2 \mathrm{~atm}$ [92], implies that genome ejection out of phage $\lambda$ into $E$. coli is a two-step mechanism. The first step, in which approximately half of the genome is ejected, is a pressure-driven process. The second step of genome internalisation could be attributed to a protein-driven ejection process (see the discussion section for a sum-up of such processes).

An example of such a two-step ejection process is the ejection of the $\mathrm{T} 5$ genome, as shown in bulk in vivo experiments [93]. After ejection of the first $8 \%$ of DNA, protein synthesis is started using this part of the genome and ejection is halted. A few minutes later the remaining part of the genome is internalised and it is suggested that one of the newly synthesised proteins pulls the DNA inside the host [94]. Another example of a two-step mechanism is the DNA ejection out of phage $\Phi 29$ [95]. In the first step, $65 \%$ of the genome is ejected into the host, probably driven by the pressure that is built up due to the DNA packaging inside the capsid. This step allows for synthesis of viral proteins, of which at least one (p17) is needed to pull the remaining part of the genome inside in the second step of internalisation. A likely explanation that not the entire DNA length is ejected by a pressuredependent mechanism is that the pressure inside the capsid drops monotonically during ejection. Thus, at a certain point, the pressure will be insufficient to eject the remaining part of the genome. The results on the ejection experiments can be understood when looking at the energy cost of packaging of the $\Phi 29$ genome [3]. It was shown that only after packaging of $\sim 40 \%$ of the genome a considerable internal force was built up inside the capsid. These results point to a clear relation between the pressures in the packaging and in the ejection process of phage $\Phi 29$.

As mentioned before, multivalent cations and polyamines can condense DNA molecules in vitro to densities comparable to those inside phage capsids [51]. This led to the suggestion that those ions will be an influential parameter in the ejection process. Bulk in vitro experiments have revealed that this is the case for ions inside the capsid, but also for those in solution. In the presence of the tetravalent cationic polyamine spermine, the pressure inside phage $\lambda$ drops significantly due to the introduction of attractive interactions between the DNA strands in the capsid. This pressure drop is evident from in vitro experiments with DNase, which digests the ejected part of the DNA [87, 89]. Light scattering experiments on bacteriophage T5 with the trivalent cationic polyamine spermidine and DNase have also shown that the pressure inside T5 capsids drops due to the presence of multivalent polyamines [96]. Under these conditions ejection is partially inhibited. However, in the absence of DNase, but in the presence of spermine, the ejection is complete for both phage T5 and phage $\lambda[89,97]$. This happens despite the spermine-induced pressure drop inside the capsid. It means that a DNA condensate outside the virus 
(which is obviously not present under conditions with DNase, as DNase digestion of the ejected genome takes place on a time scale that is faster or comparable to the rate of ejection) generates an additional force pulling on the DNA remaining inside the capsid. Figure $3 \mathrm{~b}$ shows a schematic of this process. Polyamines such as spermidine are natural multivalent cationic compounds present in bacteria and cells in millimolar concentrations, most of which associated with nucleic acids [98]. These polyamines might play a role in the final stage of in vivo DNA ejection out of phages. However, it needs to be stated that spermine is not present in E. coli [98] and that the free spermidine concentration in E. coli is lower [98] than the polyamine concentration used in these in vitro experiments.

Theoretical [46, 99, 100] and experimental [89] work has pointed out that binding of proteins to the ejected part of the genome can also have the effect of a force pulling the genome into the host. It is estimated that this force could be the dominant force at the end of ejection when the pressure has dropped significantly inside the capsid [46]. A schematic of such a system is shown in Figure 3c. Without proteins or polyamines in solution the ejection from phage would be incomplete because the last piece of DNA is unconstrained (since it does not experience any bending or repulsion forces) [89], although it would eventually diffuse out of the phage.

A system resembling more the in vivo situation is genome ejection into liposomes. A quantitative kinetic study of DNA ejection out of phage $\lambda$ into liposomes reconstituted with the membrane protein receptor LamB was performed using fluorescence microscopy [88]. These experiments showed that phage $\lambda$ genome ejection was completed within $1 \mathrm{~min}$, which is similar to the in vivo ejection rate [101]. In a different study, phage T5 DNA was injected into unilamellar liposomes that were reconstituted with the purified membrane protein receptor FhuA [102]. Toroidal structures of arbitrary shape of condensed DNA were formed in the presence of spermine, as shown by cryo-electron microscopy images. The authors stated that due to the progressive release of DNA, these structures are fundamentally different from DNA condensates formed by mixing DNA with spermine in solution.

To test whether the pressure built up inside the T5 capsid is sufficient to eject all of the genome, real time in vitro studies on single particles are performed. Those experiments revealed that, in vitro, the DNA ejection process of phage T5 predominantly takes place in a step-wise manner [4]. However, a significant amount of phages transfers the DNA into solution without halting. Ejection rates of up to $75000 \mathrm{bp} / \mathrm{s}$ are reached. The experiments are performed by tethering the phages to a surface and by hydrodynamically stretching the part of the DNA that is ejected. Fluorescent imaging is performed using an intercalating dye. The T5 genome has several sites at which the DNA exhibits single-strand interruptions in one of the DNA strands, called nicks. A correspondence was found between the position at which DNA ejection was paused and the sites where nicks occur. This suggests that the T5 nicks arrange the DNA in a certain way so as to represent an energy barrier to the ejection process. The increased flexibility of the DNA around the nick site may result in jamming of the DNA inside the connector or the tail with a consequent arrest of translocation. Thermal fluctuations could then result in a change into a favourable position to resume translocation. Such random fluctuations could also explain why sometimes a step-wise mechanism of ejection is observed and sometimes ejection seems to be continuous. Overall, the experiments on $\mathrm{T} 5$ indicate that a pressuredriven mechanism is (partially) responsible for DNA ejection, but that many questions on this mechanism remain open [34].

As the pressure in the capsid is supposed to decrease with decreasing genome length, there should be a genome length below which infection becomes less probable or impossible when a pressure-dependent mechanism is responsible for ejection. Packaging of phage $\lambda$ with genome lengths in the range of $80-105 \%$ of the wt genome length is shown to have a constant efficiency [103]. This efficiency decreases for genome lengths $>105 \%$, but those particles that are packaged are still fully infectious. Packaging also becomes less efficient for genome lengths below $80 \%$; however, not all of those particles that are packaged with such a short genome are still infectious. This is an indication that phage $\lambda$ infects cells by a pressure-dependent ejection mechanism.

One interesting counter example of pressure-driven DNA ejection is experiments on bacteriophage T7, which show evidence that a pressure built-up in its capsid is not even partially responsible for the driving mechanism of DNA delivery into host cells. Bulk in vivo measurements on the ejection of the $\mathrm{T} 7$ genome have revealed that the initiation of this process follows an enzyme-like mechanism, through which $850 \mathrm{~kb}$ of DNA is internalised [104]. The rest of the DNA is taken up through transcription by $E$. coli and, subsequently, T7 RNA polymerases, which are strong molecular motors [62]. One of the reasons for such a mechanism is probably to inhibit restriction of the viral genome, because access of type I restriction enzymes to the DNA is hindered by this transcription- 
mediated genome internalisation [105]. The transcription-independent part of the internalisation obeys a temperature dependence, which in principle would not be expected for a pressure-driven process. Instead, it shows Arrhenius kinetics similar to enzyme-driven mechanisms. It should, however, be noted that a pressure-driven ejection process can depend on temperature, as has been shown by in vitro experiments on phage T5 [96]. An explanation for this behaviour could be that there will be conformational changes of the capsid proteins interacting with the DNA, which depends on temperature. In vivo experiments on T7 also show that collapsing the membrane potential abruptly halts the process. This is an indication that the proton motive force is responsible for ejection. At the onset of infection, proteins are ejected from the virus. It is suggested that these proteins form an extensible tail functioning as a channel across the cell envelope for DNA translocation and that two of these proteins establish a molecular motor for genome internalisation [105]. Recently, the same group postulated that DNA could also be transported into the cell by water drag through the phage tail [106]. However, there is not yet experimental evidence for this.

Studies on the energy requirements of irreversible phage binding and DNA transfer into the host have shown that successful infection of phage is dependent on the presence of a membrane potential, as observed for phage T1, $\Phi 80$ and T4 [107, 108]. However, in vitro mixing of phage with their membrane receptors leads to the ejection of the genome in an energy-independent manner, as can be concluded from experiments on phage T4 [109] and phage $\lambda$ [110]. This means that, in principle, energy is not required for (initial) genome ejection, which could point to an ejection mechanism that is driven by the pressure resulting from the genome confinement in the capsid [30]. More recent experiments on the energetics of DNA transfer have shown that the transcription independent part of $\mathrm{T} 7$ genome internalisation is dependent on the presence of a membrane potential, as collapsing the membrane potential results in an abrupt halt of genome translocation [104]. Genome ejection out of $\Phi 29$ seems to be regulated by an energy-requiring protein network, because de-energisation of the cell results in a passive and less efficient entry of DNA [95]. These experiments indicate that both in vivo and in vitro experiments are needed to be performed on the same phage system to reconcile such results. Although, several mechanisms cooperate to help internalise phage DNA, the internal pressure seems to be the main mechanism responsible for the initial part of the ejection process, explaining the requirement for such high pressures inside the capsids.
Eukaryotic infecting viruses follow various mechanisms of infection and HSV-1 has developed a method that is comparable to that of dsDNA bacteriophages. After uptake by the host, HSV-1 is transported towards the nucleus via the cellular cytoskeleton $[111,112]$, where it releases its genome at the nuclear pore complex (NPC). In vitro experiments revealed that treating the HSV-1 capsid with guanidine- $\mathrm{HCl}$ results in DNA expulsion at multiple sites, supposedly the pentons [113]. These experiments indicate that the packaged genome is under pressure, but give no clue to whether a mechanism of arbitrary vertex opening is adopted in vivo. Further experiments show that capsid docking to the NPC in the presence of cytosol and energy triggers the release of DNA, which is thought to happen through one vertex region $[81,82]$. Electron microscopy images indicate that genome ejection is rapid and efficient and that eventually the empty capsid dissociates from the NPC [111,114]. Despite all these experiments, the exact mechanism of ejection out of HSV-1 remains elusive.

\section{Discussion}

The experiments on the mechanical properties of viruses have shown that the spring constant of immature $\Phi 29$ [2], MLV [7] and of empty CCMV [6] capsids are quite comparable, whereas Young's modulus of $\Phi 29$ is clearly higher than that of the other two viruses. This can be explained by the mechanism of genome encapsidation. CCMV and MLV self-assemble around the genome, but for $\$ 29$ a packaging motor is required. The packaged genome in the latter is under a high pressure ( $6 \mathrm{MPa})$, exerting a high force onto the capsid wall. This means it needs to be strong to prevent rupture and, therefore, a high Young's modulus is needed. The packaging motor of $\Phi 29$ can work against loads of up to $57 \mathrm{pN}$ [3], making it one of the strongest molecular motors known. The pressure build-up inside bacteriophage capsids as a result of DNA packaging is shown to be sufficient to initiate genome release in vitro. However, in the light of recent experimental and theoretical analysis [46, 89], the internal pressure build-up seems to be responsible only for the initial part of the genome delivery in vivo.

Several experiments point out that it is likely that phage genome ejection is a two-step process, where the first step is pressure-driven $[87,95]$ and the second step involves the genome being pulled inside by another process. This second step is likely to be any (or several) of the following: (i) transcription-based internalisation [104], (ii) enzyme-like pulling mechanisms by proteins ejected from the viral capsid [105], 
(iii) (energy-dependent) pulling by viral proteins that are synthesised after the first part of the genome is injected [95], (iv) pulling due to genome binding of proteins, independently of an external energy source $[46,89]$ and (v) pulling due to condensation of the ejected part of the genome $[89,97]$.

In vitro experiments on phages testing the hypothesis of pressure-driven DNA ejection have the big advantage that most of the parameters can be controlled precisely and that single-particle experiments are more easily monitored. However, it should be kept in mind that, in the in vivo situation, the phage remains outside the bacterium and that the DNA is injected into the host's cytoplasm. This means that normally the concentrations of the different multivalent cations and polyamines are different in the medium and inside the capsid from those inside the bacterium. These ionic compounds are able to diffuse through the capsid shell and have been shown to influence the ejection process by acting on the parts of the DNA both inside and outside the capsid [87, 89, 96, 97]. A pressuredriven ejection process is dependent on these ionic concentrations. A second point to be kept in mind is that in vitro experiments in simple buffer solutions will never be able to mimic the conditions of the bacterial cytoplasm, which resemble a highly concentrated colloidal suspension and will influence the ejection process [90].

Viral capsids are reported with many different shapes including spherical, spherocylindrical, rod-like and conical shapes. One might ask whether functionality requirements or energy minimisation during assembly can explain the existence of these different shapes. Simulations have shown that spherical shapes are more efficient in packaging and in ejecting of semiflexible polymers like DNA than ellipsoidal shapes [66]. This might explain way spherical-like shapes have evolved as the dominant shape of bacteriophage capsids. This is not in contradiction to the existence of the rod-shaped plant virus TMV as neither packaging into, nor ejecting out of, these capsids takes place. The continuum elasticity theory can explain the buckling transition of capsids from a spherical to faceted form [115], as observed during maturation of HK97 [116118] and also the existence of spherocylindrical shells [119] such as $\Phi 29$. The conical shape of the HIV-1 virus [120], however, is never stable under unconstrained energy minimisation and this shape has to be the result of physical constraints during assembly [121].

Genome packaging and ejection is dependent on capsid shape, but the presence of a non-zero capsid charge will also influence the packaging and ejection of the negatively charged DNA into and out of bacteriophage. The inner surface of the HK97 pro- capsid, for instance, is negatively charged [117]. Capsid expansion during maturation of HK97 can result from this property of the procapsid. It is proposed that, at a certain moment during packaging of the DNA, the electrostatic repulsion between the capsid and the polyelectrolyte overcomes the energy barrier for this expansion. The packaging motor needs to provide the energy to drive this process. The inner surface of the mature head remains negatively charged after the expansion is finished. This means that during ejection of the genome there will be no electrostatic attraction between the capsid and the expulsed DNA. Electrostatics also plays an important role during the self-assembly of single-stranded RNA viruses. In this case, the inner capsid surface is not negatively charged, but it has a positive charge. The assembly of the capsid around the RNA is thermodynamically favourable as the capsid proteins have an opposite charge to that of the RNA they encapsidate. Simulations show that this process is enthalpically and entropically favourable for genomes bearing a comparable but opposite charge to that of the capsid [122].

Acknowledgments. This research was supported by the Netherlands Organization for Scientific Research (NWO) through an NWOVernieuwingsimpuls grant (to G.J.L.W.), FOM-projectruimte grant (to G.J.L.W.), Swedish Research Council grant (to A.E.) and Royal Physiographic Society grant (to A.E.). W.H.R. is supported by a Rubicon grant from the Nederlandse organisatie voor Wetenschappelijk Onderzoek (NWO).

1 Bustamante, C., Macosko J. C. and Wuite, G. J. L. (2000) Grabbing the cat by the tail: manipulating molecules one by one. Nat. Rev. Mol. Cell Biol. 1, 130 - 136.

2 Ivanovska, I. L., de Pablo, P. J., Ibarra, B., Sgalari, G., MacKintosh, F. C., Carrascosa, J. L., Schmidt, C. F. and Wuite, G. J. (2004) Bacteriophage capsids: Tough nanoshells with complex elastic properties. Proc. Natl. Acad. Sci. USA 101, $7600-7605$.

3 Smith, D. E., Tans, S. J., Smith, S. B., Grimes, S., Anderson, D. L. and Bustamante, C. (2001) The bacteriophage $\Phi 29$ portal motor can package DNA against a large internal force. Nature 413, $748-752$.

4 Mangenot S., Hochrein, M., Rädler, J. and Letellier, M. (2005) Real-time imaging of DNA ejection from single phage particles. Curr. Biol. 15, 430 - 435.

5 Binnig, G., Quate, C. F. and Gerber, C. (1986) Atomic force microscope. Phys. Rev. Lett. 56, 930-933.

6 Michel, J. P., Ivanovska, I. L., Gibbons, M. M., Klug, W. S., Knobler, C. M., Wuite, G. J. L. and Schmidt, C. F. (2006) Nanoindentation studies of full and empty viral capsids and the effects of capsid protein mutations on elasticity and strength. Proc. Natl. Acad. Sci. USA 103, $6184-6189$.

7 Kol, N., Gladnikoff, M., Barlam, D., Shneck, R. Z., Rein, A. and Rousso, I. (2006) Mechanical properties of murine leukemia virus particles: Effect of maturation. Biophys. J. 91, $767-774$.

8 Carrasco, C., Carreira, A., Schaap, I. A. T., Serena, P. A., Gómez-Herrero, J., Mateu, M. G. and Pablo de, P. J. (2006) DNA-mediated anisotropic mechanical reinforcement of a virus. Proc. Natl. Acad. Sci. USA 103, 13706 - 13711.

9 Lyubchenko, Y. L., Oden, P. I., Lampner, D., Lindsay, S. M. and Dunker, K. A. (1993) Atomic force microscopy of DNA 
and bacteriophage in air, water and propanol: the role of adhesion forces. Nucleic Acids Res. 21, 1117 - 1123.

10 Anselmetti, D., Dreier, M., Lüthi, R., Richmond, T., Meyer, E., Frommer, J. and Güntherodt, H.-J. (1994) Biological materials studied with dynamic force microscopy. J. Vac. Sci. Technol. B 12, $1500-1503$.

11 Kuznetsov, Y. G., Malkin, A. J., Lucas, R. W., Plomp, M. and McPherson, A. (2001) Imaging of viruses by atomic force microscopy. J. Gen. Virol. 82, 2025-2034.

12 Plomp, M., Rice, M. K., Wagner, E. K., McPherson, A. and Malkin, A. J. (2002) Rapid visualization at high resolution of pathogens by atomic force microscopy: Structural studies of herpes simplex virus-1. Am. J. Pathol. 160, 1959 - 1966.

13 Kuznetsov, Y. G. and McPherson, A. (2006) Atomic force microscopy investigation of Turnip Yellow Mosaic Virus capsid disruption and RNA extrusion. Virology 352, 329 337.

14 Landau, L. D. and Lifshitz, E. M. (1986) Theory of Elasticity, 3rd edn., Elsevier, Oxford.

15 Klug, W. S., Bruinsma, R. F., Michel, J-P., Knobler, C. M. Ivanovska, I. L., Schmidt, C. F. and Wuite, G. J. L. (2006) Failure of viral shells. Phys. Rev. Lett. 97, 228101.

16 Howard, J. (2001) Mechanics of Motor Proteins and the Cytoskeleton. Sinauer, Sunderland, MA.

17 Douglas, T. and Young, M. (1998) Host-guest encapsulation of materials by assembled virus protein cages. Nature 393 , $152-155$.

18 Katoh, I., Yoshinaka, Y., Rein, A., Shibuya, M., Odaka, T. and Oroszlan, S. (1985) Murine leukemia virus maturation: Protease region required for conversion from "immature" to "mature" core form and for virus infectivity. Virology 145 $280-292$.

19 Yoshinaka, Y. and Luftig, R. B. (1977) Murine leukemia virus morphogenesis: Cleavage of $\mathrm{P} 70$ in vitro can be accompanied by a shift from a concentrically coiled internal strand ("immature") to a collapsed ("mature") form of the virus core. Proc. Natl. Acad. Sci. USA 74, 3446 - 3450.

20 Yeager, M., Wilson-Kubalek, E. M., Weiner, S. G., Brown, P. O. and Rein, A. (1998) Supramolecular organization of immature and mature murine leukemia virus revealed by electron cryo-microscopy: Implications for retroviral assembly mechanisms. Proc. Natl. Acad. Sci. USA 95, 7299 - 7304.

21 Tama, F. and Brooks, C. L. (2005) Diversity and identity of mechanical properties of icosahedral viral capsids studied with elastic network normal mode analysis. J. Mol. Biol. 345 , $299-314$.

22 Hespenheide, B. M., Jacobs, D. J. and Thorpe, M. F. (2004) Structural rigidity in the capsid assembly of cowpea chlorotic mottle virus. J. Phys. Condens. Matter 16, S5055-S5064.

23 Zandi, R. and Reguera, D. (2005) Mechanical properties of viral capsids. Phys. Rev. E 72, 021917.

24 Falvo, M. R., Washburn, S., Superfine, R., Finch, M., Brooks, F. P. Jr., Chi, V. and Taylor, R. M. II (1997) Manipulation of individual viruses: Friction and mechanical properties. Biophys. J. 72, $1396-1403$.

25 Knez, M., Sumser, M. P., Bittner, A. M., Wege, C., Jeske, H., Hoffmann, D. M. P., Kuhnke, K. and Kern, K. (2004) Binding the tobacco mosaic virus to inorganic surfaces. Langmuir 20, $441-447$

26 Teschke, C. M., McGough, A. and Thuman-Commike, P. A. (2003) Penton release from P22 heat-expanded capsids suggests importance of stabilizing penton-hexon interactions during capsid maturation. Biophys. J. 84, 2585-2592.

27 Anderson, T. F., Rappaport, C. and Muscatine, N. A. (1953) On the structure and osmotic properties of phage particles. Ann. Inst. Pasteur 84, 5- 15.

28 Cordova, A., Deserno, M., Gelbart, W. M. and Ben-Shaul, A (2003) Osmotic shock and the strength of viral capsids. Biophys. J. 85, $70-74$.

29 Purohit, P. K., Kondev, J. and Phillips, R. (2003) Mechanics of DNA packaging in viruses. Proc. Natl. Acad. Sci. USA 100, $3173-3178$
30 Earnshaw, W. C. and Casjens, S. R. (1980) DNA packaging by the double-stranded DNA bacteriophages. Cell 21,319-331.

31 Bazinet, C. and King, J. (1985) The DNA translocating vertex of dsDNA bacteriophage. Annu. Rev. Microbiol. 39, 109 129.

32 Black, L. W. (1989) DNA Packing in dsDNA bacteriophages. Annu. Rev. Microbiol. 43, 267-292.

33 Moore, S. D. and Prevelige, P. E. Jr. (2002) DNA Packaging: A new class of molecular motors. Curr. Biol. 12, R96-R98.

34 Ponchon, L., Mangenot, S., Boulanger, P. and Letellier, L. (2005) Encapsidation and transfer of phage DNA into host cells: From in vivo to single particles studies. Biochim. Biophys. Acta 1724, $255-261$.

35 Kainov, D. E., Tuma, R. and Mancini, E. J. (2006) Hexameric molecular motors: P4 packaging ATPase unravels the mechanism. Cell. Mol. Life Sci. 63, 1095-1105.

36 Kaiser, D. and Masuda, T. (1973) In vitro assembly of bacteriophage lambda heads. Proc. Natl. Acad. Sci. USA 70, $260-264$

37 Hohn, B. and Hohn, T. (1974) Activity of empty, headlike particles for packaging of DNA of bacteriophage $\lambda$ in vitro. Proc. Natl. Acad. Sci. USA 71, 2372 - 2376.

38 Hohn, B. (1975) DNA as substrate for packaging into bacteriophage lambda, in vitro. J. Mol. Biol. 98, 93 - 106.

39 Bjornsti, M., Reilly, B. E. and Anderson, D. L. (1981) In vitro assembly of the Bacillus subtilis bacteriophage $\Phi 29$. Proc. Natl. Acad. Sci. USA 78, $5861-5865$.

40 Guo, P., Grimes, S. and Anderson, D. (1986) A defined system for in vitro packaging of DNA-gp3 of the Bacillus subtilis bacteriophage $\Phi 29$. Proc. Natl. Acad. Sci. USA 83, 3505 3509.

41 Lee, C-S. and Guo, P. (1995) In vitro assembly of infectious virions of double-stranded DNA phage $\Phi 29$ from cloned gene products and synthetic nucleic acids. J. Virol. 69, 5018-5023.

42 Gaussier, H., Yang, Q. and Catalano, C. E. (2006) Building a virus from scratch: Assembly of an infectious virus using purified components in a rigorously defined biochemical assay system. J. Mol. Biol. 357, 1154-1166.

43 Odijk, T. (1998) Hexagonally packed DNA within bacteriophage T7 stabilized by curvature stress. Biophys. J. 75, 1223 1227.

44 Tzlil, S., Kindt, J. T., Gelbart, W. M. and Ben-Shaul, A. (2003) Forces and pressures in DNA packaging and release from viral capsids. Biophys. J. 84, 1616-1627.

45 Riemer, S. C. and Bloomfield, V. A. (1978) Packaging of DNA in bacteriophage heads: Some considerations on energetics. Biopolymers 17, 785 - 794.

46 Inamdar, M. M., Gelbart, W. M. and Phillips, R. (2006) Dynamics of DNA ejection from bacteriophage. Biophys. J. $91,411-420$.

47 Guo, P., Peterson, C. and Anderson, D. (1987) Prohead and DNA-gp3-dependent ATPase activity of the DNA packaging protein gp16 of bacteriophage Ф29. J. Mol. Biol. 197, 229 236.

48 Earnshaw, W. C. and Harrison, S. C. (1977) DNA arrangement in isometric phage heads. Nature 268, $598-602$.

49 Rau, D. C., Lee, B. and Parsegian, V. A. (1984) Measurement of the repulsive force between polyelectrolyte molecules in ionic solution: Hydration forces between parallel DNA double helices. Proc. Natl. Acad. Sci. USA 81, 2621 - 2625.

50 Schellman, J. A. and Parthasarathy, N. (1984) X-ray diffraction studies on cation-collapsed DNA. J. Mol. Biol. 175, 313 329.

51 Bloomfield, V. A. (1997) DNA condensation by multivalent cations. Biopolymers 44, $269-282$.

52 Cerritelli, M. E., Cheng, N., Rosenberg, A. H., McPherson, C. E., Booy, F. P. and Steven, A. C. (1997) Encapsidated conformation of bacteriophage T7 DNA. Cell 91, 271-280.

53 Rau, D. C. and Parsegian, V. A. (1992) Direct measurements of the intermolecular forces between counterion-condensed DNA double helices; Evidence for long range attractive hydration forces. Biophys. J. 61, 246 - 259. 
54 Evilevitch, A., Castelnovo, M., Knobler, C. M. and Gelbart, W. M. (2004) Measuring the force ejecting DNA from phage. J. Phys. Chem. B 108, $6838-6843$.

55 Robinson, M. A., Wood, J. P. A., Capaldi, S. A., Baron, A. J., Gell, C., Smith, D. A. and Stonehouse N. J. (2006) Affinity of molecular interactions in the bacteriophage \$29 DNA packaging motor. Nucleic Acids Res. 34, 2698-2709.

56 Lee, T. J. and Guo, P. (2006) Interaction of gp16 with pRNA and DNA for genome packaging by the motor of bacterial virus phi29. J. Mol. Biol. 356, 589-599.

57 Moll, W. D. and Guo, P. (2005) Translocation of nicked but not gapped DNA by the packaging motor of bacteriophage phi29. J. Mol. Biol. 351, 100-107.

58 Simpson, A. A., Tao, Y., Leiman, P. G., Badasso, M. O., He, Y., Jardinek, P. J., Olson, N. H., Morais, M. C., Grimes, S., Anderson, D. L., Baker, T. S. and Rossmann, M. G. (2000) Structure of the bacteriophage $\Phi 29$ DNA packaging motor. Nature 408, 745 - 750 .

59 Guasch, A., Joan Pous, J., Ibarra, B., Gomis-Rüth, F. X. Valpuesta, J. M., Sousa, N., Carrascosa, J. L. and Coll, M. (2002) Detailed architecture of a DNA translocating machine: The high-resolution structure of the bacteriophage Ф29 connector particle. J. Mol. Biol. 315, $663-676$.

60 Xiang, Y., Morais, M. C., Battisti, A. J., Grimes, S., Jardine, P. J., Anderson, D. L. and Rossmann, M. G. (2006) Structural changes of bacteriophage $\Phi 29$ upon DNA packaging and release. EMBO J. 25, 5229-5239.

61 Ashkin, A. (1970) Acceleration and trapping of particles by radiation pressure. Phys. Rev. Lett. 24, $156-159$.

62 Wang, M. D., Schnitzer, M. J., Yin, H., Landick, R., Gelles, J. and Block, S. M. (1998) Force and velocity measured for single molecules of RNA polymerase. Science 282, $902-907$.

63 Wuite, G. J. L., Smith, S. B., Young, M., Keller, D. and Bustamante, C. (2000) Single-molecule studies of the effect of template tension on T7 DNA polymerase activity. Nature 404 $103-106$.

64 Svoboda, K. and Block, S. M. (1994) Force and velocity measured for single kinesin molecules. Cell 77, 773 - 784

65 Mehta, A. D., Rock, R. S., Rief, M., Spudich, J. A., Mooseker, M. S. and Cheney, R. E. (1999) Myosin-V is a processive actinbased motor. Nature 400, $590-593$

66 Ali, I., Marenduzzo, D. and Yeomans, J. M. (2006) Polymer packaging and ejection in viral capsids: Shape matters. Phys. Rev. Lett. 96, 208102

67 Chemla, Y. R., Aathavan, K., Michaelis, J., Grimes, S. Jardine, P. J., Anderson, D. L. and Bustamante, C. (2005) Mechanism of force generation of a viral DNA packaging motor. Cell 122, 683-692.

68 Maluf, N. K., Gaussier, H., Bogner, E., Feiss, M. and Catalano, C. E. (2006) Assembly of bacteriophage lambda terminase into a viral DNA maturation and packaging machine. Biochemistry 45, 15259 - 15268.

69 Leiman, P. G., Kanamaru, S., Mesyanzhinov, V. V., Arisaka, F. and Rossmann,M. G. (2003) Structure and morphogenesis of bacteriophage T4. Cell. Mol. Life Sci. 60, 2356-2370.

70 Casjens, S., Wyckoff, E., Hayden, M., Sampson, L., Eppler, K., Randall, S., Moreno, E. T. and Serwer, P. (1992) Bacteriophage P22 portal protein is part of the gauge that regulates packing density of intravirion DNA. J. Mol. Biol. 224, 1055 - 1074.

71 Lander, G. C., Tang, L., Casjens, S. R., Gilcrease, E. B. Prevelige, P., Poliakov, A., Potter, C. S., Carragher, B. and Johnson, J. E. (2006) The structure of an infectious P22 virion shows the signal for headful DNA packaging. Science 312 , $1791-1795$

72 Hendrix, R. W. (1978) Symmetry mismatch and DNA packaging in large bacteriophages. Proc. Natl. Acad. Sci. USA 75 $4779-4783$

73 Tao, Y., Olson, N. H., Xu, W., Anderson, D. L., Rossmann, M G. and Baker, T. S. (1998) Assembly of a tailed bacterial virus and its genome release studied in three dimensions. Cell 95 , $431-437$.
74 Baumann, R. G., Mullaney, J. and Black, L. W. (2006) Portal fusion protein constraints on function in DNA packaging of bacteriophage T4. Mol. Microbiol. 61, 16-32.

75 Maluf, N. K. and Feiss, M. (2006) Virus DNA translocation: progress towards a first ascent of Mount Pretty Difficult. Mol. Microbiol. 61, 1 - 4.

76 Serwer, P. (2003) Models of bacteriophage DNA packaging motors. J. Struct. Biol. 141, 179-188.

77 Beard, P. M., Duffy, C. and Baines, J. D. (2004) Quantification of the DNA cleavage and packaging proteins UL15 and UL28 in A and B capsids of herpes simplex virus type 1. J. Virol. 78, $1367-1374$.

78 Homa, F. L. and Brown, J. C. (1997) Capsid assembly and DNA packaging in herpes simplex virus. Rev. Med. Virol. 7, 107-122.

79 Sheaffer, A. K., Newcomb, W. M., Gao, M., Yu, D., Weller, S. K., Brown, J. C. and Tenney, D. J. (2001) Herpes simplex virus DNA cleavage and packaging proteins associate with the procapsid prior to its maturation. J. Virol. 75, 687-698.

80 Booy, F. P., Newcomb, W. W., Trus, B. L., Brown, J. C., Baker, T. S. and Steven, A. C. (1991) Liquid-crystalline, phage-like packing of encapsidated DNA in herpes simplex virus. Cell 64, $1007-1015$

81 Ojala, P. M., Sodeik, B., Ebersold, M. W., Kutay, U. and Helenius, A. (2000) Herpes simplex virus type 1 entry into host cells: Reconstitution of capsid binding and uncoating at the nuclear pore complex in vitro. Mol. Cell. Biol. 20, 49224931.

82 Greber, U. F. and Fassati, A. (2003) Nuclear import of viral DNA genomes. Traffic 4, $136-143$.

83 Baker, M. L., Jiang, W., Rixon, F. J. and Chiu, W. (2005) Common ancestry of herpesviruses and tailed DNA bacteriophages. J. Virol. 79, 14967 - 14970.

84 McGeoch, D. J., Rixon, F. J. and Davison, A. J. (2006) Topics in herpesvirus genomics and evolution. Virus Res. 117, 90104.

85 Hershey, A. D. and Chase, M. (1952) Independent functions of viral protein and nucleic acid in growth of bacteriophage. J. Gen. Physiol. 36, 39 - 56.

86 Purohit, P. K., Inamdar, M. M., Grayson, P. D., Squires, T. M., Kondev, J. and Phillips, R. (2005) Forces during bacteriophage DNA packaging and ejection. Biophys. J. 88, 851 - 866.

87 Evilevitch, A., Lavelle, L., Knobler, C. M. Raspaud, E. and Gelbart, W. M. (2003) Osmotic pressure inhibition of DNA ejection from phage. Proc. Natl. Acad. Sci. USA 100, $9292-$ 9295.

88 Novick, S. L. and Baldeschwieler, J. D. (1988) Fluorescence measurement of the kinetics of DNA injection by bacteriophage $\lambda$ into liposomes. Biochemistry 27, 7919-7924.

89 Evilevitch, A. (2006) Effects of condensing agent and nuclease on the extent of ejection from phage. J. Phys. Chem. B. 110, $22261-22265$

90 Castelnovo, M., Bowles, R. K., Reiss, H. and Gelbart, W. M. (2003) Osmotic force resisting chain insertion in a colloidal suspension. Eur. Phys. J. E 10, 191 - 197.

91 Evilevitch, A., Gober, J. W., Phillips, M., Knobler, C. M. and Gelbart, W. M. (2005) Measurements of DNA lengths remaining in a viral capsid after osmotically suppressed partial ejection. Biophys. J. 88, $751-756$.

92 Grayson, P., Evilevitch, A., Inamdar, M. M., Purohit, P. K., Gelbart, W. M., Knobler, C. M. and Phillips, R. (2006) The effect of genome length on ejection forces in bacteriophage lambda. Virology 348, $430-436$.

93 Lanni ,Y. T. (1968) First-step-transfer deoxyribonucleic acid of bacteriophage T5. Bacteriol. Rev. 32, 227 - 242.

94 Letellier, L., Boulanger, P., Frutos de, M. and Jacquot, P. (2003) Channeling phage DNA through membranes: from in vivo to in vitro. Res. Microbiol. 154, 283-287.

95 González-Huici, V., Salas, M. and Hermoso, J. M. (2004) The push-pull mechanism of bacteriophage Ø29 DNA injection. Mol. Microbiol. 52, 529-540. 
96 Frutos de, M., Letellier, L. and Raspaud, E. (2005a) DNA Ejection from Bacteriophage T5: Analysis of the kinetics and energetics. Biophys J. 88, 1364 - 1370.

97 Frutos de, M., Brasiles, S., Tavares, P. and Raspaud, E. (2005b) Effect of spermine and DNase on DNA release from bacteriophage T5. Eur. Phys. J. E 17, 429 - 434.

98 Igarashi, K. and Kashiwagi, K. (2000) Polyamines: Mysterious modulators of cellular functions. Biochem. Biophys Res. Commun. 271, $559-564$.

99 Sung, W. and Park, P. J. (1996) Polymer translocation through a pore in a membrane. Phys. Rev. Lett. 77, $783-787$.

100 Zandi, R., Reguera, D., Rudnick, J. and Gelbart, W. M. (2003) What drives the translocation of stiff chains? Proc. Natl Acad. Sci. USA 100, 8649 - 8653.

101 Garcia, L. R. and Molineux, I. J. (1995) Rate of translocation of bacteriophage T7 DNA across the membranes of Escherichia coli. J. Bacteriol. 177, 4066 - 4076.

102 Lambert, O., Letellier, L., Gelbart, W. M. and Rigaud, J.-L. (2000) DNA delivery by phage as a strategy for encapsulating toroidal condensates of arbitrary size into liposomes. Proc. Natl. Acad. Sci. USA 97, $7248-7253$.

103 Feiss, M., Fisher, R. A., Crayton, M. A. and Egner, C. (1977) Packaging of the bacteriophage $\lambda$ chromosome: Effect of chromosome length. Virology 77, $281-293$.

104 Kemp, P., Gupta, M. and Molineux, I. J. (2004) Bacteriophage T7 DNA ejection into cells is initiated by an enzyme-like mechanism. Mol. Microbiol. 53, 1251 - 1265 .

105 Molineux, I. J. (2001) No syringes please, ejection of phage T7 DNA from the virion is enzyme driven. Mol. Microbiol. 40, $1-8$.

106 Molineux, I. J. (2006) Fifty-three years since Hershey and Chase; much ado about pressure but which pressure is it? Virology 344, 221-229.

107 Hancock, R. E. W. and Braun, V. (1976) Nature of the energy requirement for the irreversible adsorption of bacteriophages T1 and $\Phi 80$ to Escherichia coli. J. Bacteriol. 125, $409-415$.

108 Kalasauskaite, E. and Grinius, L. (1979) The role of energyyielding ATPase and respiratory chain at early stages of bacteriophage T4 infection. FEBS Lett. 99, 287 - 291

109 Jesaitis, M. A. and Goebel, W. F. (1955) Lysis of T4 phage by the specific lipocarbohydrate of phase II Shigella Sonnei. J. Exp. Med. 102, 733 - 752.

110 Randall-Hazelbauer, L. and Schwartz, M. (1973) Isolation of the bacteriophage lambda receptor from Escherichia coli. J. Bacteriol. 116, 1436 - 1446.
111 Sodeik, B., Ebersold, M. W. and Helenius, A. (1997) Microtubule-mediated transport of incoming herpes simplex virus 1 capsids to the nucleus. J. Cell Biol. 136, 1007-1021.

112 Radtke, K., Döhner, K. and Sodeik, B. (2006) Viral interactions with the cytoskeleton: a hitchhiker's guide to the cell. Cell. Microbiol. 8, 387-400.

113 Newcomb, W. W. and Brown, J. C. (1994) Induced Extrusion of DNA from the capsid of herpes simplex virus type 1. J. Virol. 68, 433 - 440.

114 Batterson, W., Furlong, D. and Roizman, B. (1983) Molecular genetics of herpes simplex virus VIII. Further characterization of a temperature-sensitive mutant defective in release of viral DNA and in other stages of the viral reproductive cycle. J. Virol. 45, $397-407$.

115 Lidmar, J., Mirny, L. and Nelson, D. R. (2003) Virus shapes and buckling transitions in spherical shells. Phys. Rev. E 68, 051910.

116 Lata, R., Conway, J. F., Cheng, N., Duda, R. L., Hendrix, R. W., Wikoff, W. R., Johnson, J. E., Tsurata, H. and Steven, A. (2000) Maturation dynamics of a viral capsid: Visualization of transitional intermediate states. Cell 100, 253-263.

117 Conway, J. F., Wikoff, W. R., Cheng, N., Duda, R. L., Hendrix, R. W., Johnson, J. E. and Steven, A. C. (2001) Virus maturation involving large subunit rotations and local refolding. Science 292, $744-748$.

118 Gan, L., Conway, J. F., Firek, B. A., Cheng, N., Hendrix, R. W., Steven, A. C., Johnson, J. E. and Duda, R. L. (2004) Control of crosslinking by quaternary structure changes during bacteriophage HK97 maturation. Mol. Cell 14, 559 569.

119 Nguyen, T. T., Bruinsma, R. F. and Gelbart, W. M. (2005) Elasticity theory and shape transitions of viral shells. Phys. Rev. E 72, 051923.

120 Ganser-Pornillos, B. K., Schwedler von, U. K., Stray, K. M., Aiken, C. and Sundquist, W. I. (2004) Assembly properties of the human immunodeficiency virus type $1 \mathrm{CA}$ protein. J. Virol. 78, 2545-2552.

121 Nguyen, T. T., Bruinsma, R. F. and Gelbart, W. M. (2006) Continuum theory of retroviral capsids. Phys. Rev. Lett. 96, 078102.

122 Angelescu, D. G., Bruinsma, R. and Linse, P. (2006) Monte Carlo simulations of polyelectrolytes inside viral capsids. Phys. Rev. E 73, 041921.

\section{To access this journal online: http://www.birkhauser.ch/CMLS}

